\title{
Structural Characterization of Calcium Sulfate Bone Graft Substitute Cements
}

\author{
Evangelos P. Favvas ${ }^{a, b} *$, Konstantinos L. Stefanopoulos ${ }^{a}$, Nikolaos Ch. Vordos ${ }^{b}$, George I. Drosos ${ }^{c}$, \\ Athanasios Ch. Mitropoulos ${ }^{b}$ \\ ${ }^{a}$ Membranes and Materials for Environmental Separations Laboratory, Institute of Nanoscience and \\ Nanotechnology, NCSR “Demokritos”, 153 41, Agia Paraskevi, Attica, Greece \\ ${ }^{b}$ Hephaestus Laboratory, Department of Petroleum and Mechanical Engineering, Eastern Macedonia \\ and Thrace Institute of Technology, 654 04, St. Lucas, Cavala, Greece \\ ${ }^{c}$ Department of Orthopaedic Surgery, Medical School, Democritus University of Thrace, University \\ General Hospital of Alexandroupolis, 681 00, Dragana, Alexandroupolis, Greece
}

Received: November 5, 2015; Revised: June 07, 2016; Accepted: July 28, 2016

\begin{abstract}
The aim of this work was to study the structural characteristics of commercially available bone graft substitute (BGS) ceramic cements. In particular, the microstructure of two calcium sulfate cements was investigated. For this purpose, nitrogen and mercury porosimetry, $\mathrm{X}$-ray diffraction (XRD) and scanning electron microscopy (SEM) measurements have been carried out. Mercury intrusion porosimetry results revealed that the structural characteristics of the two samples varied significantly. These structural differences can be justified when compared with their compression and bending strength properties. As a result, a proper understanding of microstructure of BGS materials is crucial in the search of what is optimal for bone regeneration.
\end{abstract}

Keywords: biomaterials, bone graft substitutes (BGS), ceramics, porosimetry, structural properties

\section{Introduction}

Bone graft substitute (BGS) cements have been widely used in fracture treatment in various fixation augmentation techniques aiming at increasing implant stability in the mechanically weak bone. The first documented bone-grafting procedure was carried out during 1668 by the Dutch surgeon Job van Meek'ren using dog cranium xenograft while Fred Albee, first described autologous bone grafting in 1915, using part of the tibia for spinal fusion ${ }^{1}$. Calcium phosphate cements have found many clinical applications for repair of bone defects and bone augmentation because of their biocompatible and biodegradable characteristics ${ }^{2}$. On the other hand, calcium sulfate cements have also been investigated as alternative candidates to autograft in the restoration of bone defect $^{3}$. It is worth mentioning that calcium sulfate $\left(\mathrm{CaSO}_{4}\right)$ has been used as bone void filler for over 100 years and has been extensively researched and thoroughly reviewed by several investigators. In 1892, Dreesmann reported results of using calcium sulfate to fill osseous cavities in humans with tuberculosis ${ }^{4,5}$. Among calcium sulfate-based bioceramics the $a$-Calcium sulfate hemihydrate $(\mathrm{CSH})$ powder $\left(\mathrm{CaSO}_{4}\right.$ $1 / 2 \mathrm{H}_{2} \mathrm{O}$ ) is very popular as bone substitute in clinical fields ${ }^{6}$. Furthermore, calcium sulfate substitutes occupy a unique position in the group of regenerative materials and are recognized as safe and bioactive implant materials. They have been successfully used in bone substitution although they have been criticized for their rapid resorption. Calcium sulfate cements, especially calcium sulfate dihydrate, CSD,

*e-mail: e.favvas@inn.demokritos.gr
$\left(\mathrm{CaSO}_{4} \cdot 2 \mathrm{H}_{2} \mathrm{O}\right)$, as well as the derivative of the calcium sulfate hemihydrate, $\mathrm{CSH},\left(\mathrm{CaSO}_{4} \cdot 0.5 \mathrm{H}_{2} \mathrm{O}\right)$ after the mixing of the powder $\mathrm{CSH}$ with water, have long been used for filling bone defects because both their capability for bone repair and their excellent biocompatibility ${ }^{7,8}$. To this end, CSD continues to be the object of research and interest as one of the most successful bone cements ${ }^{9}$, because it has i) the ability to undergo in situ setting after filling the defects, ii) has a good biocompatibility without inducing an inflammatory response and iii) promotes bone healing ${ }^{10}$.

In a previous work ${ }^{11}$, the mechanical performance of two different calcium phosphate cements, two different calcium sulphate cements, one nanocrystalline hydroxyapatite and one polymethylmethacrylate (PMMA) BGS ceramic cements have been tested. Among all those tested materials the calcium sulfate substitutes were the best performing specimens in bending strength and they had also exhibited good compressive strength values. Based on this criterion, the calcium sulfate samples were the materials of choice for the present study. We utilized a variety of experimental techniques such as porosimetry (nitrogen and mercury) for revealing their structural architecture from nano- to micrometer scale, diffraction for the evaluation of the crystalline phase, and electron microscopy (SEM) for providing the surface and/or crystal features. Our aim is to investigate how the structural characteristics of the BGS are related with their mechanical properties. Indeed, the results suggest that the pore structure plays a very important role in the mechanical performance of the substitutes. 


\section{Experimental}

The two BGS calcium sulfate cements, CS1 and CS2, have been provided by Wright Medical Technology Inc., Arlington, USA. Both biomaterials were prepared strictly following the specified manufacturers' instructions and then, as-mixed immediately inserted in specially designed and built moulding devices in order to produce all necessary specimens according to ISO 5833. CS1 and CS2 were purchased as two packet components, one powder and one liquid. CS1 was produced by mixing each component for 1 min while CS2 components were mixed for $30 \mathrm{~s}$ in vacuum mixer (see ref. 11 for more details). The specific surface area (SSA), the porosity and the pore size of the samples have been measured using commercial nitrogen (Quantachrome Autosorb-1, with MP upgrade) and mercury (Quantachrome Autoscan 25 and Autoscan 60) porosimetries. Before measurements, the samples were degassed overnight at $90^{\circ} \mathrm{C}$ in a vacuum of $10^{-7}$ Torr. Further, XRD (Bruker D8 advance x-ray diffraction) measurements were performed and SEM (Jeol JSM 7401F Field Emission) images were obtained for revealing the crystallographic and macro-microscopic characteristics of the samples.

\section{Results and Discussion}

The nitrogen adsorption technique is widely used for detecting open pores mainly in the micro- mesopore region. According to IUPAC classification ${ }^{12}$, micropores have size $D_{p}<2 \mathrm{~nm}$, mesopores $\left(2<D_{p}<50 \mathrm{~nm}\right)$ and macropores $\left(D_{p}>50 \mathrm{~nm}\right)$. The specific surface area (SSA) was determined from the adsorption isotherms by the BET method ${ }^{13}$. The pore volume, $V_{p}$, was calculated from the adsorbed mass near saturation using the density of liquid nitrogen at $77 \mathrm{~K}\left(0.808 \mathrm{~g} / \mathrm{cm}^{3}\right)$ and assuming that pores are filled subsequently with condensed adsorbate in the normal liquid state. Finally, the average pore size distribution was deduced according to BJH method ${ }^{14}$. The micropore volume was estimated according to DR (Dubinin-Radushkevich) plots ${ }^{15}$. The DR plots showed that both samples exhibit negligible microporosity. Figure 1 illustrates the nitrogen adsorption isotherms at $77 \mathrm{~K}$ for CS1 and CS2 samples. According to IUPAC ${ }^{12}$, both isotherms are close to type III corresponding to non-porous or macroporous solids. They also exhibit $\mathrm{H} 3$ hysteresis loop along the mesoporous region which is caused by the existence of non-rigid aggregates of plate-like particles or assemblages of slit-shaped pores ${ }^{16}$. In particular, the obtained nitrogen adsorption $(77 \mathrm{~K})$ isotherms are typical of samples with slit-shaped mesopores that extended into the macropore region, caused by the spaces between the plates of material ${ }^{17}$. It is noteworthy that that no significant differences are observed between the two isotherms.

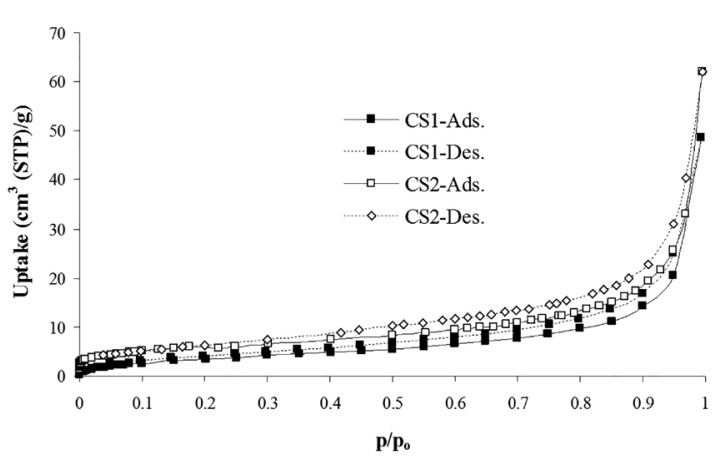

Figure 1: Nitrogen adsorption isotherms at $77 \mathrm{~K}$ for CS1 and CS2 samples.

Table 1 presents the calculated structural parameters of the samples. The low total adsorbed amounts of both samples result in very small mesopore volumes.

Table 1: Structural parameters obtained from nitrogen porosimetry measurements; $S$ is the SSA, $V_{p}$ is the mesopore pore volume and $D_{p}$ is the average pore size.

\begin{tabular}{lccc}
\hline Sample & $\begin{array}{c}S \\
\left(\mathrm{~m}^{2} / \mathrm{g}\right)\end{array}$ & $\begin{array}{c}V_{p} \\
\left(\mathrm{~cm}^{3} / \mathrm{g}\right)\end{array}$ & $\begin{array}{c}D_{p} \\
(\mathrm{~nm})\end{array}$ \\
\hline $\mathrm{CS} 1$ & 13 & 0.03 & 3.7 \\
$\mathrm{CS} 2$ & 20 & 0.05 & 3.7 \\
\hline
\end{tabular}

The specific surface area is about 13 and $20 \mathrm{~m}^{2} / \mathrm{g}$ for CS1 and SC2 samples respectively while the average pore size is approximately $3.7 \mathrm{~nm}$ for both samples. We have also attempted to calculate the micropore volume, according to DR plots. The results, however, showed that both samples exhibit negligible microporosity. In general, the absence of micropores could be significant for improvement of the mechanical properties of the BGS cements. In conclusion, the nitrogen porosimetry results suggest a limited mesopore network in both samples.

Mercury porosimetry is a useful tool for determining the pore structure of solids with pore sizes varying from large mesopores to macropores. The equivalent pore radius $r$ is deduced according to the capillary pressure Washburn equation $^{18}$ :

$$
r=\frac{2 \gamma \cos \Theta}{P_{c}}[\mathrm{~m}]
$$

where $P_{c}$ is the capillary pressure [Pa], $\gamma$ the interfacial tension $[\mathrm{N} / \mathrm{m}],\left(\gamma_{\mathrm{H} g / \mathrm{ar}}=0.471 \mathrm{~N} / \mathrm{m}\right)$ and $\Theta$ the wetting angle $\left(\Theta_{\mathrm{Hg} / \mathrm{air}}=\right.$ $\left.140^{\circ}\right)$.

Figures 2 and 3 show the high-pressure mercury intrusion curves and the calculated pore size distributions for the SC1 and SC2 samples. Obviously the pore structure of the two samples differs significantly in a size range between 0.05 and $2 \mu \mathrm{m}$. In particular, the values of the pore volume and the 


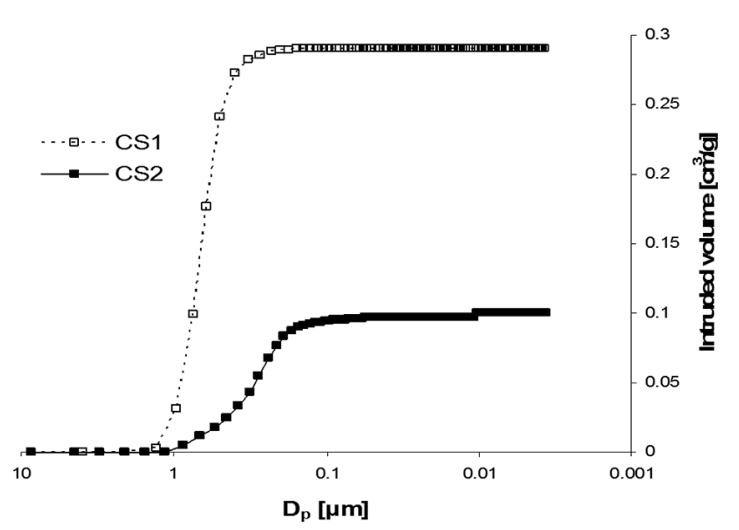

Figure 2: Mercury intrusion curves. The lowest pore sizes correspond to the maximum pressure.

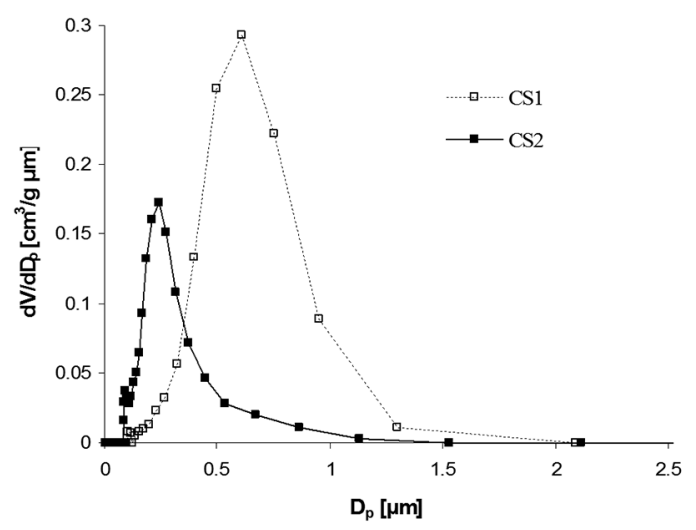

Figure 3: Pore size distribution of CS1 and CS2 calcium sulfate cements.

pore size of the CS1 sample are about three times larger to those of CS2 sample. In addition the porosity is much higher for CS1 sample (43\%) as compared with CS2 (20\%) (see also Table 2). These structural changes could be explained in terms of recipe differences such as sintering temperature or sintering program ${ }^{19}$. On the other hand, low-pressure measurements (extending to pore sizes up to $180 \mu \mathrm{m}$ ) of both samples did not reveal any significant structural changes. Similar results have also been observed in a series of cements containing CSD crystals and TiHA particles ${ }^{20}$. In addition, a bimodal pore size distribution has been deduced because of the void formation between CSD plate-like crystals and the cavity existence between TiHA grain aggregates. In the case of the pristine CSD crystal, however, a single pore size distribution $\left(D_{p}\right)$ has been observed and the structural characteristics of that sample $\left(\varepsilon=37 \%, D_{p}=0.55 \mu \mathrm{m}\right)$ are very close to CS1 (Table 2).
Figure 4 shows the XRD patterns of the two ceramics. Both studied samples have identical crystallographic structure and their patterns were identified with the CSD $\left(\mathrm{CaSO}_{4} \cdot 2 \mathrm{H}_{2} \mathrm{O}\right)$ characteristic peaks ${ }^{21,22}$. According to the pdf file (33-311), based on the Crystallographica Search-Match (CSM) program produced by the International Centre for Diffraction Data $(I C D D)$, both materials are described as monoclinic crystal systems and the lattice parameters are: a: 6.2845 , b: 15.2079 and c: $5.6776 \AA$.

They are also crystalline although their microstructure and the preparation route are different, as previously discussed. It is worth mentioning that high crystallinity has also been observed in biphasic calcium phosphate ceramics $(\mathrm{BCP})^{23}$. In another study, however, calcium phosphate cements either with or without addition of without the addition of $\mathrm{NaHCO}_{3}$ to the cement powder exhibited low crystallinity ${ }^{24}$.

Figure 5 illustrates the SEM micrographs of the CSD crystals. Plate-like crystal structure is the common characteristic shape of both CSD samples with an average particle size $(\mu \mathrm{m})$ of about $2 \times 2 \times 6$. According to Figure 5 the crystalline sizes of the two calcium sulfate cements extend from 0.5 up to about $6 \mu \mathrm{m}$ in length. In addition, the thickness of the flake-like particles is about 1.5-1.9 $\mu \mathrm{m}$. Further, the crystallites locate at a random orientation and the formatted inter-grains voids inhere in the macroporous classification.

One may note that these empty-space macropore voids are necessary for the growth of the new physical bone after the implantation of the substitute into the patient. This coalescing interfacial zone of the CSD substitute crystals provides a scaffold for bone-cell adhesion and further bone ingrowth ${ }^{25}$. Overall this crystalline structure is common for CSD bone cements as reported in a plurality of relevant works ${ }^{26}$.

One of the most important characteristics of this type of materials is the mechanical stability. In a previous work ${ }^{11}$, the studied materials were tested in compression and bending strength and were also compared with a polymethylmethacrylate (PMMA) sample, often referred as the reference material. Table 3 presents the calculated values for each biomaterial, expressed as average \pm standard deviation (for more details, see ref. 11).

The results show that CS2 has better mechanical properties compared to CS1. Mercury porosimetry results confirm this finding. Indeed, CS1 has larger values for pore volume, porosity and pore size distribution compared to those of CS2. On the other hand, both tested samples failed under loads lower than those of PMMA. In particular, in compression CS1 and CS2 showed a strength value of approximately $11 \%$ and $60 \%$ of PMMA respectively. Further, in bending strength

Table 2: Characteristic properties obtained from mercury porosimetry measurements; $\varepsilon$ is the porosity.

\begin{tabular}{|c|c|c|c|c|}
\hline Sample & $\begin{array}{c}S \\
\left(\mathrm{~m}^{2} / \mathrm{g}\right)\end{array}$ & $\begin{array}{c}V_{p} \\
\left(\mathrm{~cm}^{3} / \mathrm{g}\right)\end{array}$ & $\begin{array}{c}\varepsilon \\
(\%)\end{array}$ & $\begin{array}{c}D_{p} \\
(\mu \mathrm{m})\end{array}$ \\
\hline CS1 & 1.9 & 0.29 & 43 & 0.61 \\
\hline $\mathrm{CS} 2$ & 2.6 & 0.10 & 20 & 0.24 \\
\hline
\end{tabular}


CS1 and CS2 exhibited a strength value of approximately $11 \%$ and $22 \%$ of PMMA respectively. It is then obvious that the structural properties of ceramic materials affect their mechanical performance. In specific, the reduction in mechanical strength of CS1, mainly due to the increase of

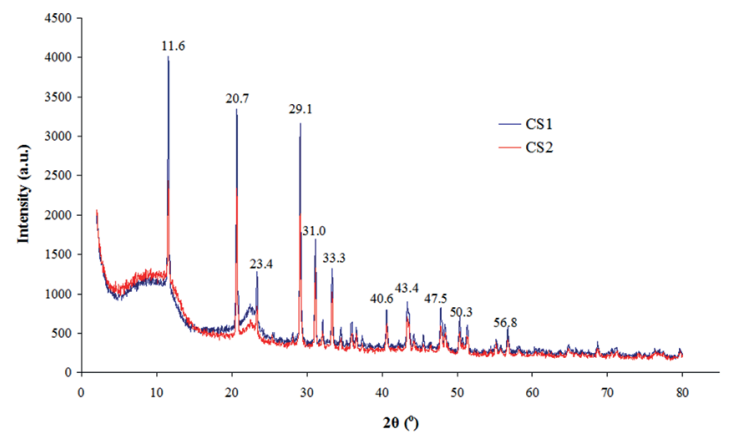

Figure 4: XRD patterns of the two studied samples. the void volume, might be critical for regeneration in loadbearing bones. On the other hand, the increase of the porosity could concentrate more proteins and therefore stimulate inducible cells in soft issues to form inductive bone ${ }^{23}$. To this end, the pore structure, the mechanical strength as well as the bioresorbability are crucial parameters for the optimal BGS selection.

\section{Conclusions}

The structural architecture of two calcium sulfate cements was investigated by nitrogen and mercury porosimetry, $\mathrm{XRD}$ and SEM. The nitrogen adsorption isotherms suggest negligible microporosity and a limited mesopore network at both samples. Further, the XRD measurements revealed that the tested samples have similar crystallographic structure and their patterns were identified with the CSD $\left(\mathrm{CaSO}_{4} \cdot 2 \mathrm{H}_{2} \mathrm{O}\right)$ characteristic peaks. SEM micrographs
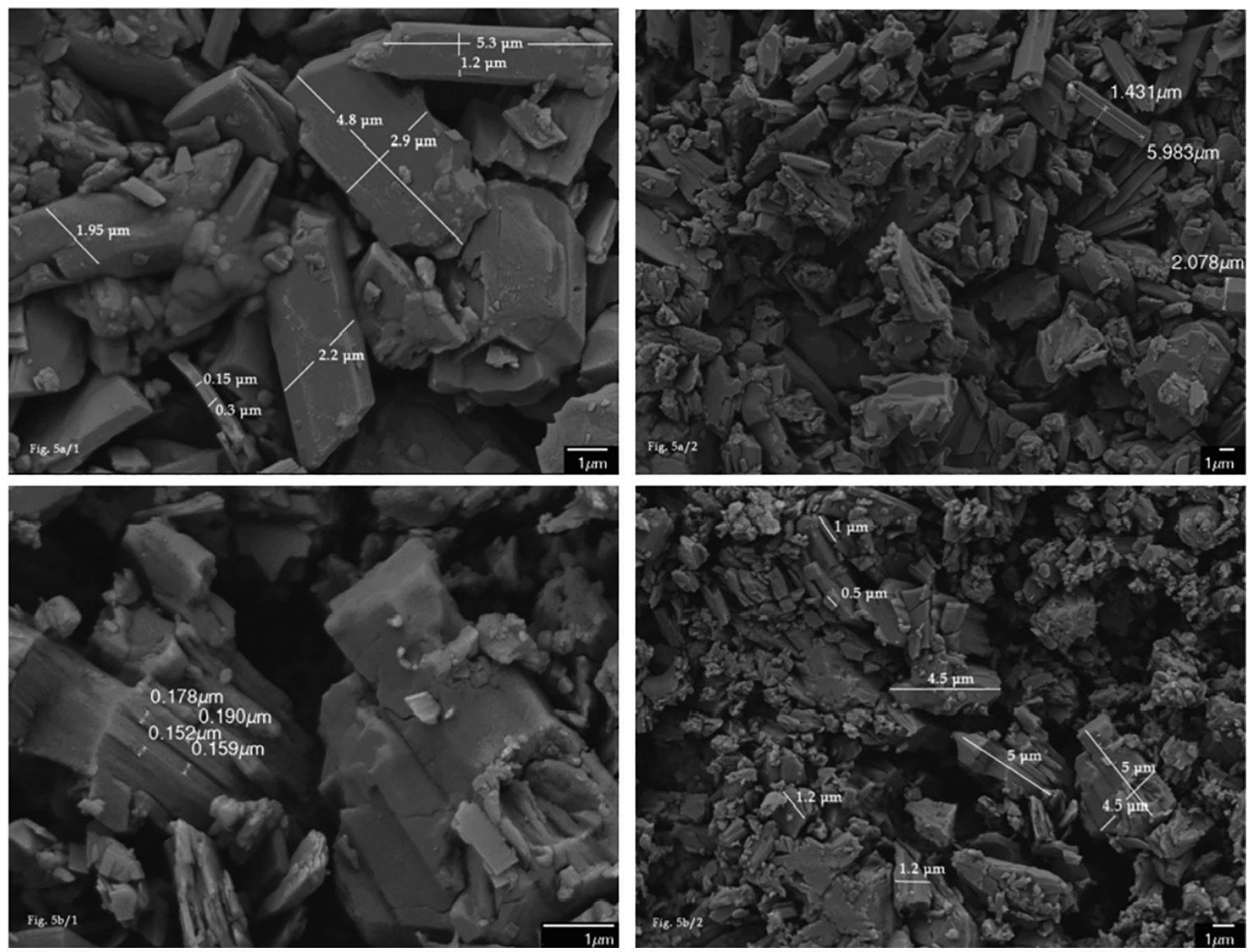

Figure 5: SEM images for CS1 sample (up) and CS2 sample (down).

Table 3: Compressive and bending strength for CS1, CS2 and a PMMA BGS.

\begin{tabular}{lcc}
\hline Sample & Compression strength aver. \pm st. dev. (MPa) & Bending strength aver. \pm st.dev. (MPa) \\
\hline PMMA & $88.5 \pm 0.8$ & $54.8 \pm 7.4$ \\
CS1 & $9.9 \pm 1.6$ & $6.1 \pm 1.1$ \\
CS2 & $52.9 \pm 3.6$ & $11.9 \pm 3.4$ \\
\hline
\end{tabular}


showed that crystallites locate at a random orientation and plate-like crystal structure is the common characteristic shape of both samples. The mercury porosimetry measurements revealed significant structural differences of the samples in pore sizes ranging between 0.05 and $2 \mu \mathrm{m}$. These differences in the pore structure can be fully justified in comparison with their compression and bending strength performance. As a result, the reduction in mechanical strength of CS1, mainly due to the increase of porosity, might be critical for regeneration in load-bearing bones. On the other hand, the increase of the pore volume favors the concentration of higher amount of proteins which could improve osteoinductivity. In conclusion, a better understanding of the effect of intrinsic material properties on biological performance, mechanical strength and biodegradability of BSG substitutes might be proved to be helpful in the search of what is optimal for bone regeneration.

\section{Acknowledgements}

The present work is a result in the framework of NSRF. The Nanocapillary Project (Thales Framework, MIS 375233) of the Eastern Macedonia and Thrace Institute of Technology is co-financed by Greece and the European Union in the frame of operational program "Education and lifelong learning investing in knowledge society", Greek Ministry of Education and Religious Affairs, Culture and Sports, NSRF 2007-2013.

\section{References}

1. Jahangir AA, Nunley RM, Mehta S, Sharan A; Washington Health Policy Fellows. Bone-graft substitutes in orthopaedic surgery. AAOS Now. 2008;2:1-5.

2. del Valle S, Miño N, Muñoz F, González A, Planell JA, Ginebra MP. In vivo evaluation of an injectable Macroporous Calcium Phosphate Cement. Journal of Materials Science: Materials in Medicine. 2007;18(2):353-361.

3. Calori GM, Mazza E, Colombo M, Ripamonti C. The use of bone-graft substitutes in large bone defects: any specific needs? Injury. 2011;42 Suppl 2:S56-63.

4. Peltier LF. The use of plaster of Paris to fill defects in bone. Clinical Orthopaedics. 1961;21:1-31.

5. Peters CL, Hines JL, Bachus KN, Craig MA, Bloebaum RD. Biological effects of calcium sulfate as a bone graft substitute in ovine metaphyseal defects. Journal of Biomedical Materials Research. Part A. 2006;76A(3):456-462.

6. Nilsson M, Wang JS, Wielanek L, Tanner KE, Lidgren L. Biodegradation and biocompatability of a calcium sulphatehydroxyapatite bone substitute. Journal of Bone and Joint Surgery. British. 2004;86(1):120-125.

7. Bahn SL. Plaster: a bone substitute. Oral Surgery, Oral Medicine, and Oral Pathology. 1966;21(5):672-681.
8. Chen Z, Liu H, Liu X, Lian X, Guo Z, Jiang HJ, et al. Improved workability of injectable calcium sulfate bone cement by regulation of self-setting properties. Materials Science and Engineering: C. 2013;33(3):1048-1053.

9. Jepegnanam TS, von Schroeder HP. Rapid Resorption of Calcium Sulfate and Hardware Failure Following Corrective Radius Osteotomy: 2 Case Reports. The Journal of Hand Surgery. 2012;37(3):477-480.

10. Coetzee AS. Regeneration of Bone in the Presence of Calcium Sulfate. Archives of otolaryngology (Chicago, Ill.: 1960). 1980;106(7):405-409.

11. Drosos GI, Babourda E, Magnissalis EA, Giatromanolaki A, Kazakos K, Verettas DA. Mechanical characterization of bone graft substitute ceramic cements. Injury. 2012;43(3):266-271.

12. Sing KSW, Everett DH, Haul RAW, Moscou L, Pierotti RA, Rouquérol J, et al. Reporting physisorption data for gas/solid systems with special reference to the determination of surface area and porosity (Recommendations 1984). Pure and Applied Chemistry. 1985;57(4):603-619.

13. Brunauer S, Emmet PH, Teller EJ. Adsorption of Gases in Multimolecular Layers. Journal of the American Chemical Society. 1938;60(2):309-319.

14. Barret EP, Joyner LG, Halenda PP. The Determination of Pore Volume and Area Distributions in Porous Substances. I. Computations from Nitrogen Isotherms. Journal of the American Chemical Society. 1951;73(1):373-380.

15. Dubinin MM, Radushkevich LV. Equation of the Characteristic Curve of Activated Charcoal. Proceedings of the Academy of Sciences, Physical Chemistry Section, USSR. 1947;55:331-333.

16. Thommes M. Physical Adsorption Characterization of Nanoporous Materials. Chemie Ingenieur Technik. 2010;82(7):1059-1073.

17. Rouquerol F, Rouquerol J, Sing KSW. Adsorption by Powders and Porous Solids: Principles, Methodology and Applications. San Diego: Academic Press; 1999.

18. Washburn EW. Note on a Method of Determining the Distribution of Pore Sizes in a Porous Material. Proceedings of the National Academy of Sciences of the United States of America. 1921;7(4):115-116.

19. Hannink G, Arts JJ. Bioresorbability, porosity and mechanical strength of bone substitutes: what is optimal for bone regeneration? Injury. 2011;42 Suppl 2:S22-S25.

20. Ślósarczyk A, Czechowska J, Paszkiewicz Z, Zima A. New bone implant material with calcium sulfate and Ti modified hydroxyapatite. Journal of Achievements in Materials and Manufacturing Engineering. 2010;43(1):170-177.

21. Huan Z, Chang J. Self-setting properties and in vitro bioactivity of calcium sulfate hemihydrate-tricalcium silicate composite bone cements. Acta Biomaterialia. 2007;3(6):952-960.

22. Morris MC, McMurdie HF, Evans EH, Paretzkin B, Parker HS, Panagiotopoulos NC. Standard X-ray Diffraction Powder Patterns. Washington: National Bureau of Standards; 1981; $110 \mathrm{p}$. 
23. Li X, Liu H, Niu X, Fan Y, Feng Q, Cui FZ, et al. Osteogenic differentiation of human adipose-derived stem cells induced by osteoinductive calcium phosphate ceramics. Journal of Biomedical Materials Research. Part B, Applied Biomaterials. 2011;97(1):10-19.

24. del Real RP, Wolke JGC, Vallet-Regí M, Jansen JA. A new method to produce macropores in calcium phosphate cements. Biomaterials. 2002;23(17):3673-3680.
25. Mirzayan R, Panossian V, Avedian R, Forrester DM, Menendez LR. The use of calcium sulfate in the treatment of benign bone lesions. A preliminary report. The Journal of bone and joint surgery. American. 2001;83-A(4):355-358.

26. Pan Z, Lou Y, Yang G, Ni X, Chen M, Xu H, et al. Preparation of calcium sulfate dihydrate and calcium sulfate hemihydrate with controllable crystal morphology by using ethanol additive. Ceramics International. 2013;39(5):5495-5502. 